\title{
Prognostic modeling of transformer aging using Bayesian particle filtering
}

\author{
V. M. Catterson \\ Institute for Energy and Environment, University of Strathclyde, \\ Glasgow, United Kingdom. \\ Email: v.m.catterson@strath.ac.uk
}

\begin{abstract}
The goal of condition monitoring is to accurately assess the current health of an asset, in order to generate a prognosis, i.e. predict its remaining useful life. In the absence of a fault which causes premature failure, transformer degradation is linked to paper aging. Research and experience have resulted in models of paper aging where hotspot temperature is the key driver. However, these deterministic equations give a false sense of certainty about remaining insulation life.

This paper demonstrates the use of Bayesian particle filtering for transformer life prognostics. This technique allows quantification of the uncertainties surrounding aspects such as the initial degree of polymerization of the paper, the relationship between hotspot temperature and measurands, and the accuracy of measurements. A case study from an in-service 180 MVA transformer is used to illustrate its potential.
\end{abstract}

\section{INTRODUCTION}

Within the power industry, there is a growing need and desire for prognostic systems. While diagnostic systems can identify the current health of an asset, a prognostic system makes a prediction about future behaviour and equipment remaining useful life (RUL) [1]. As much power network infrastructure approaches or reaches the end of its design life, there is a growing need for tools to support the prioritisation of refurbishment and repair, with reasonable forewarning of impending failure.

However, a prediction of RUL is inherently uncertain, since it is based on assumptions about future environmental and operational conditions, and their impact on asset deterioration. The prognostic system should be able to incorporate this uncertainty, preferably explicitly, and still make predictions which are useful for decision-making.

Power transformers are critical for network operation, and can be very costly to replace. At the moment, maintenance decision-making is performed manually taking account of hours in service, operating conditions, and the results of any diagnostics such as dissolved gas analysis or thermal monitoring [2]. Good prognostic tools would allow repair and replacement to be planned to extract maximum operational life, while minimizing the risk of a failure in service.

One tool is given in the IEEE C57.91 Loading Guide [3]. This standard contains a model for the rate of transformer aging, based on hotspot temperature. However, this model has the disadvantage that it is deterministic, which affects the result in two ways. First, it gives an exact figure for the rate of aging for a given hotspot temperature, without taking account of the probabilistic nature of deterioration. Secondly, the calculation of hotspot temperature from other measurands does not allow incorporation of systematic or random sensor inaccuracy.

This paper introduces Bayesian particle filtering [4] as an approach to modeling and managing the uncertainty inherent in predicting future asset health. The aim is to construct powerful prognostic systems which can truly support engineers in decision-making, by explicitly including probabilistic factors. A case study of particle filtering as applied to transformer aging is used to demonstrate how the technique represents current asset state, and how it makes predictions of RUL. This can be used to generate predictions for multiple future scenarios, such as repeated weekly load pattern versus an overload.

\section{TRANSFORMER AGING}

Transformer end of life is governed by the aging of paper insulation [5]. Specifically, heat leads to polymerization of the cellulose chains, causing the paper to become more brittle over time. If no action is taken, the tensile strength of the paper drops to such a level that it cannot withstand network faults and other stresses, and insulation integrity is lost.

The life of the transformer can therefore be quantified by the degree of polymerization (DP) of the paper at its most aged point. Since the rate of aging is determined primarily by temperature [3], aging is most rapid at the transformer hotspot, and the paper here will have the lowest DP of the transformer. New paper has a DP approximately in the range of 1000-1200 [6], while end of life is generally considered to be 200 [5].

A model for paper aging is given in IEEE standard C57.91 [3]. This assumes a life of 150,000 hours at a temperature of $110^{\circ} \mathrm{C}$ for a standard transformer to reach a DP of 200. The standard defines an aging acceleration factor, $F_{A A}$, based on hotspot temperature, $\Theta_{H}$, which is greater or less than 1 for temperatures above or below $110^{\circ} \mathrm{C}$ respectively:

$$
F_{A A}=e^{\frac{15000}{383}-\frac{15000}{273+\Theta_{H}}}
$$

This model is given as a guide for transformer loading. Since overloading increases temperature, and thus the aging acceleration factor, the duration and size of an overload has implications for transformer life. However, the deterministic 
nature of the model gives a false sense of certainty about the effects of an overload on aging. That is, a given temperature above $110^{\circ} \mathrm{C}$ is always expected to consume paper life at exactly the rate determined by the equation above.

In reality, uncertainties surround many factors, such as the precise life consumption at a given temperature, the initial DP of the paper, and the accuracy of measuring temperature and load. A probabilistic modeling approach which can account for these uncertainties would give a better, more accurate view of remaining transformer life.

\section{BAYESIAN PARTICLE FILTERING}

Bayesian particle filtering is a method of estimating current state, and simulating future state of a Markovian system (where the system state depends only on its previous state and current conditions) [4]. Each 'particle' represents a possible system state at time $t-1$, from which can be simulated a likely state at time $t$. By running multiple simulations (particles) in parallel, with probabilistically varied initial conditions and state transitions, the technique can generate a probability density function over all particle end of life conditions. This gives a range of possible lifetimes, weighted by the probability of their occurrence.

The system model comprises two parts: the process model $f$ and the measurement model $h$ [7]:

$$
\begin{aligned}
& x_{t}=f\left(x_{t-1}, u_{t}\right) \\
& y_{t}=h\left(x_{t}, v_{t}\right)
\end{aligned}
$$

for $x_{t}$ system state at time $t, y_{t}$ measurements at time $t, u$ and $v$ noise terms. The process model represents the underlying aging process of the system under study, while the measurement model captures the relationship between any measurements made and the true state of the system.

At each timestep, two calculations are made for each particle $i$ :

1) Simulation of the new system state $x_{t}^{i}$, given previous system state $x_{t-1}^{i}$ :

$$
x_{t}^{i}=f\left(x_{t-1}^{i}, u_{t}\right) \forall i
$$

2) Weighting of particle likelihood, given the probability of new measurement values occurring if this particle represents the true system state $p\left(y_{t} \mid x_{t}^{i}\right)$, combined with this particle's weight at the previous timestep $w_{t-1}^{i}$ :

$$
w_{t}^{i}=p\left(y_{t} \mid x_{t}^{i}\right) \times w_{t-1}^{i} \forall i
$$

Prognostic capability comes from the first of these, as it predicts the next timestep. The second performs a diagnosis, using measurements to adjust the certainty of each particle representing the true current state of the system. Longer horizon life predictions are made by repeatedly calculating equation 4 for subsequent timesteps $t+1, t+2, \ldots$.

Bayesian particle filtering has been used in fields where mechanical aging and fatigue are the dominant failure modes [8], [9]. However, the technique is applicable to any process which can be represented as Markovian.

\section{PARTICle FILTERING FOR TRANSFORMER AGING}

Transformer aging can be considered a partially-observable Markov process, where the state of the paper cannot be directly observed, but measurands like top oil temperature and load give some insight into the degradation rate. The process is Markovian, since the state of the paper depends only on its previous state and current conditions. The aging acceleration factor equation [3] can be rewritten as a recurrence relation with this property, giving the process model:

$$
l_{t}=l_{t-1}-\exp \left(\frac{15000}{383}-\frac{15000}{\left(\Theta_{H_{t}}+273\right)}\right)+u_{t}
$$

where $l_{t}$ is remaining useful life (RUL) at time $t, \Theta_{H_{t}}$ is hotspot temperature at time $t$, and $u_{t}$ is process noise. The uncertainties in this model include the initial condition $l_{0}$ (that is, initial hours of life due to initial DP); parameters of the equation linking hotspot to measurands; and the noise (i.e. slight variation in how much life is consumed for a given hotspot temperature).

The measurement model is also extracted from [3]. Since the hotspot temperature is not measured directly, $\Theta_{H}$ must be inferred from other parameters. Given the ratio of measured load current to rated load, $K$, and measured top oil temperature, $\Theta_{t o}$, the measurement model is:

$$
\Theta_{H}=\Theta_{t o}+\left(80-\Delta \Theta_{t o / a, R}\right) \times K^{2 m}
$$

where $m$ and $\Delta \Theta_{t o / a, R}$ are transformer-specific parameters. The $m$ is related to the cooling mode of the transformer $(0.8$ for ONAN and ONAF), and $\Delta \Theta_{t o / a, R}$ is the difference in temperature between top oil and ambient at rated current.

\section{CASe Study}

A $180 \mathrm{MVA}, 275 / 132 \mathrm{kV}$ transmission transformer in the UK was chosen for study. This transformer had been in service for 39 years at the start of this study, and was typically very lightly loaded due to its location on the network (20\% on average). The data available was ambient temperature, top oil temperature, and load current, recorded hourly over one whole year, representing the transformer's 40th year in service.

The year was considered typical in terms of ambient and loading conditions and outages, and therefore it was assumed that the profile of life consumption during this year was the same as that over all 39 years in service.

Assumptions were made about the sensors and paper initial conditions, described below, but it should be noted that with more detailed information about the specific performance of the sensors used and the supply of paper, these assumptions could be refined, thus increasing the fidelity of the simulation.

The particle filter was initialized with 100 particles equally weighted. Initial life of the paper for each particle was drawn from a Gaussian distribution with mean 150,000 hrs and standard deviation $50 \mathrm{hrs}$. Process error (variation on the amount of life consumed at each timestep) was assumed to be Gaussian with a standard deviation of $10^{-6}$. Errors on 
measurements were assumed to follow a Gaussian distribution, with top oil temperature having a standard deviation of $0.5^{\circ} \mathrm{C}$, and load current having a standard deviation of $10 \mathrm{~A}$. All errors were assumed to have a mean of zero.

The following sections describe how the particle filter performs diagnosis with historical normal operating data, and prognosis of predicted future conditions.

\section{A. Normal operating conditions}

As the data was recorded hourly, the simulation timestep was set to one hour. At each timestep, the next values of load current and top oil temperature were read, and for each particle equation 6 was used to update the RUL values. Next, equation 7 was used to update the particle likelihood weights. Finally, the weights were normalized so that:

$$
\sum^{i} w_{t}^{i}=1
$$

These stages were repeated for all 8,760 hours in the year, and the yearly data was repeated 40 times to simulate the full life in service.

Figure 1 shows the probability density function (PDF) of remaining transformer life at both the start and the end of the 40 year simulation. This is generated by performing kernel density estimation [10] on the initial (red) and final (blue) life values of all particles $l^{i}$, weighted by the particle weights $w^{i}$.

The peak of the PDF curve represents the most likely RUL value, i.e. a majority of highly weighted simulations report a similar outcome. This is illustrated most clearly by the red curve of the initial RUL values, which were drawn from a Gaussian distribution centered on 150,000 hrs. The mean value of the red curve is very close to this center (149,994 hrs).

Notably, very little aging of the paper has taken place, as represented by the mean and median values of each PDF (Table I). This is due to the generally light loading on the transformer and mild climate in the UK. The maximum

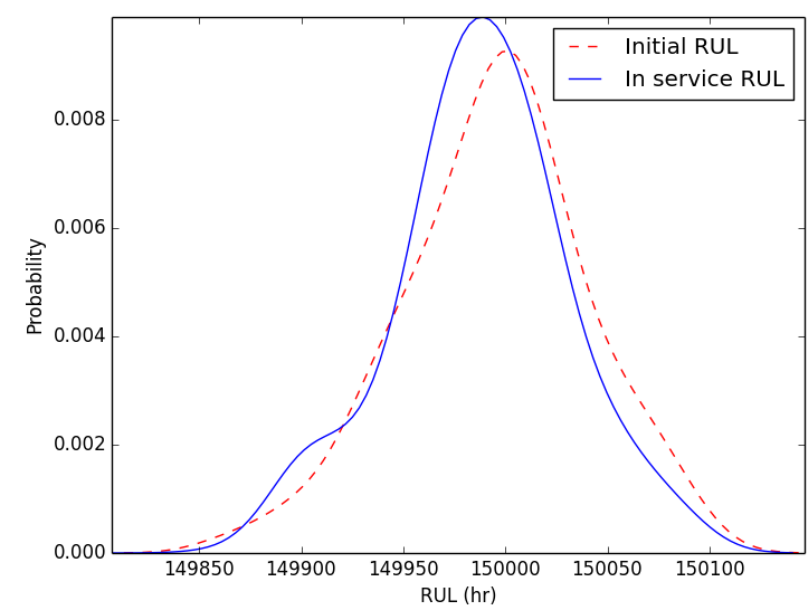

Fig. 1. Change in remaining life of transformer
TABLE I

STATISTICAL PROPERTIES OF INITIAL AND FINAL RUL PDFS

\begin{tabular}{|c|c|c|}
\hline PDF & Mean (hr) & Median (hr) \\
\hline Initial RUL & 149,994 & 149,997 \\
\hline Final RUL & 149,989 & 149,991 \\
\hline
\end{tabular}

loading did not exceed 0.5 per unit, and averaged around 0.2 p.u.; while the peak ambient temperature was $35^{\circ} \mathrm{C}$ with a mean around $14^{\circ} \mathrm{C}$. This suggests that the dominant failure mode of this unit is not likely to be aging.

It can be seen that the shape of the curve changes between initial conditions and the end of the simulation. While the red curve is a good approximation of the original Gaussian distribution that RUL values were drawn from, the blue curve is skewed towards the left. This is to be expected, since the life of the transformer paper can only be consumed and not restored. The simulations are therefore biased towards consuming life, with the resulting skew to shorter RUL values.

\section{B. Prognosis under different loading}

The previous section shows how the particle filter was used to determine a probabilistic representation of transformer RUL for its time in service. This section demonstrates how to convert this diagnosis into a prognosis of future RUL, under different loading conditions.

Prediction with the particle filter is as simple as running the simulation with predicted measurement data instead of actual measurements. In this case, the loading and top oil temperature must be predicted for the prognostic period.

For this study, three different loading scenarios for year 41 of the transformer's life were considered.

- Case 1: The transformer experiences similar conditions to the previous year. Load and temperature data are predicted to be the same as the year of measured data.

- Case 2: A doubling of loading due to significant construction of commercial properties in the area. Load is predicted to double while retaining the same weekly pattern. Temperature data is predicted to be the same as the previous year.

- Case 3: Network reconfiguration due to an unexpected transformer failure on a nearby circuit. Load is expected to begin as in the previous year, sharply increase to $120 \%$ of rated current for 6 hours, fall to a maximum of about $90 \%$ for 8 months, then return to previous levels. Ambient temperature is predicted to be the same as the previous year.

A section of these different load profiles is shown in Figure 2, at the initiation of case 3's six hour overload.

Running the particle filter with these three scenarios gives the result shown in Figure 3. It can be seen that cases 1 and 2 give very similar curves with about three hours of difference between them, showing that there is very little difference in aging. This is because even with a doubling of load, the daily maximum is still only around 0.6 p.u., and the rate of aging is relatively low. 


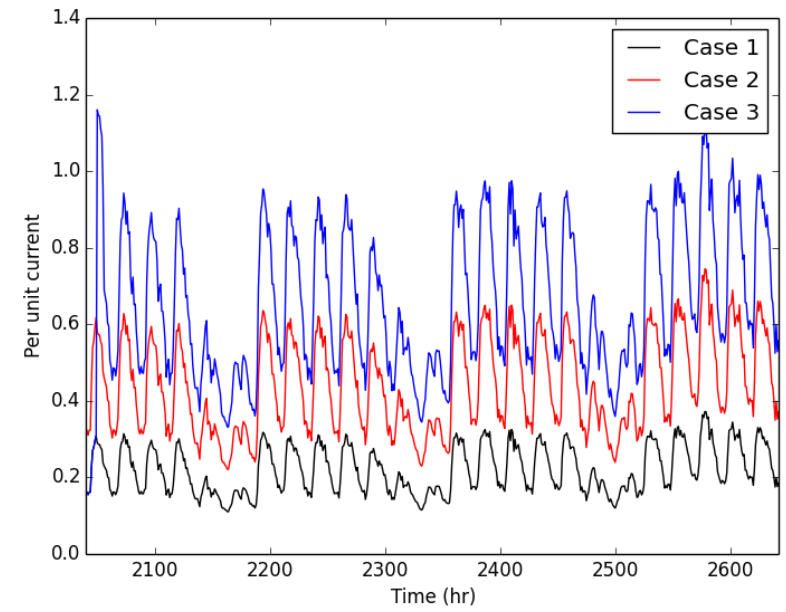

Fig. 2. Part of predicted loading scenarios

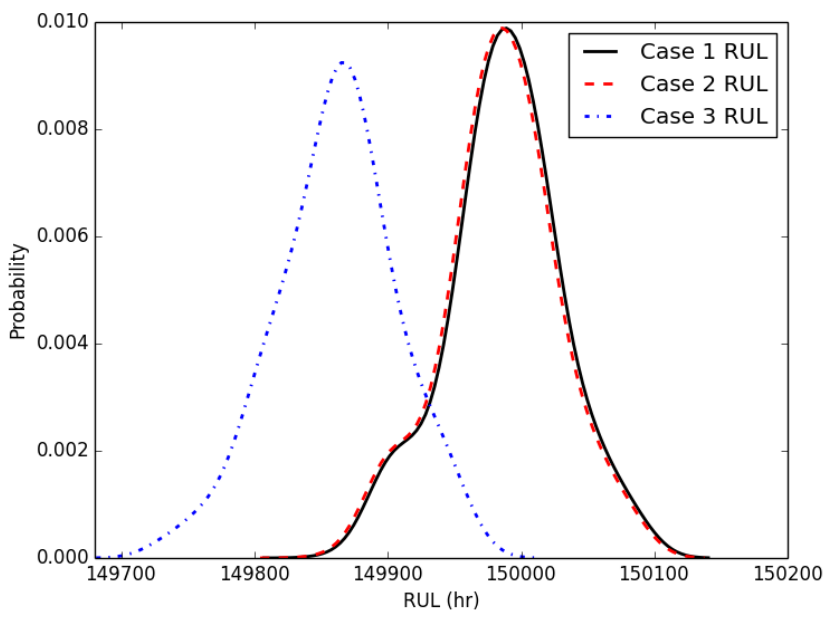

Fig. 3. RUL at the end of three scenarios

Case 3, which contains a six hour overload, displays a much larger decrease in RUL. The overload plus the higher loading combines to reduce RUL by approximately 150 hours, compared to case 1 . This is still relatively minor aging overall, since the periods of peak loading are restricted to a few hours each day, with weekend and night time load generally below 0.6 p.u.

While this study examined the impact of different loading scenarios, the same type of investigation can be applied to ambient temperature conditions. Hotter conditions than normal may be expected to affect hotspot temperature and thus aging. By simulating a number of possible temperature profiles, various scenarios can be examined.

\section{CONCLUSIONS}

Bayesian particle filtering is a method of probabilistic simulation of a system where a number of parameters are unknown or uncertain. This can be used for asset health prognostics by predicting the future environmental and operating conditions of the asset, and simulating how that scenario will affect deterioration. The two key strengths of the technique are that the output is the likelihood of various outcomes (and not one deterministic result), and that uncertainty about both the measurement system and the underlying process are modelled explicitly.

Within this paper, the use of particle filtering to track and predict transformer aging was shown. Standard equations for paper aging were rearranged into recurrence relation form, and measurements of load current and top oil temperature were assumed to be noisy. From this basis, the in-service life of a case study transformer was simulated, showing very little aging due to light loading and mild ambient conditions.

Three future loading scenarios were predicted, and their effect on simulated lifetime examined. The generation of a PDF for each scenario means that the particle filter outputs not only the most likely outcome given the circumstances (the peak value on the curve), but also the range of possible outcomes with their corresponding likelihood. This makes particle filtering an effective tool for predicting future asset health, to assist with maintenance decision-making.

Future work could integrate other transformer data. For example, some designs of transformer incorporate sacrificial paper strips within the tank, which can be removed one by one over time and the true state of the paper measured directly. This information could be used to reset the particle filter to a known DP and hence RUL, with correspondingly more accurate results. Further, additional failure modes such as partial discharge could be modeled, extending the tool to predict RUL in the presence of one or more fault mechanisms.

\section{REFERENCES}

[1] A. K. S. Jardine, D. Lin, and D. Banjevic, "A review on machinery diagnostics and prognostics," Mechanical Systems and Signal Processing, vol. 20, 2006.

[2] T. K. Saha, "Review of Modern Diagnostic Techniques for Assessing Insulation Condition in Aged Transformers," IEEE Trans. Dielectr. Electr. Insul., vol. 10, no. 5, pp. 903-917, Oct. 2003.

[3] IEEE Power and Energy Society, "IEEE Std. C57.91 IEEE Guide for Loading Mineral-Oil-Immersed Transformers and Step-Voltage Regulators," 2011.

[4] J. Liu and M. West, "Combined parameter and state estimation in simulation-based filtering," in Sequential Monte Carlo Methods in Practice, N. J. G. De Freitas, Ed., 2001.

[5] H.-Z. Ding and Z. D. Wang, "Kinetic analysis and modelling of the ageing process for kraft paper aged in natural ester dielectric fluid," in 2006 IEEE Conf. Electrical Insulation and Dielectric Phenomena, Oct. 2006, pp. 505-508.

[6] A. Van Schijndel, P. A. A. F. Wouters, and J. M. Wetzer, "Modeling of replacement alternatives for power transformer populations," IEEE Trans. Power Delivery, vol. 27, no. 2, pp. 506-513, Apr. 2012.

[7] M. S. Arulampalam, S. Maskell, N. Gordon, and T. Clapp, "A tutorial on particle filters for online nonlinear/non-Gaussian Bayesian tracking," IEEE Trans. Signal Processing, vol. 50, no. 2, pp. 174-188, Feb. 2002.

[8] M. E. Orchard and G. J. Vachtsevanos, "A particle-filtering approach for on-line fault diagnosis and failure prognosis," Trans. Inst. Measurement and Control, vol. 31, no. 3, pp. 221-246, 2009.

[9] S. Butler and J. Ringwood, "Particle filters for remaining useful life estimation of abatement equipment used in semiconductor manufacturing," in Conf. Control and Fault Tolerant Systems, Oct. 2010.

[10] J. S. Kim and C. Scott, "Robust kernel density estimation," in IEEE Int. Conf. Acoustics, Speech and Signal Processing, Mar. 2008, pp. 33813384 . 